\title{
Patchwork Reconstruction with Resolution Modeling for Digital Breast Tomosynthesis
}

\author{
Koen Michielsen, ${ }^{*}$ Katrien Van Slambrouck, and Johan Nuyts \\ Department of Imaging and Pathology, 83 Medical Imaging Research Center, KU Leuven, Belgium
}

Anna Jerebko

Siemens Healthcare, Erlangen, Germany

(Dated: January 7, 2013)

\begin{abstract}
Purpose: Digital breast tomosynthesis is a relatively new diagnostic x-ray modality that allows high resolution breast imaging while suppressing interference from overlapping anatomical structures. However, proper visualization of micro-calcifications remains a challenge. For the subset of systems we consider, the main cause of deterioration is movement of the x-ray source during exposures. We propose a modified grouped coordinate ascent algorithm that includes a specific acquisition model to compensate for this deterioration.
\end{abstract}

Methods: We create a resolution model based on the movement of the x-ray source during image acquisition and combine this with a grouped coordinate ascent algorithm. Choosing planes parallel to the detector surface as the groups enables efficient implementation of the position dependent resolution model. In the current implementation, the resolution model is approximated by a Gaussian smoothing kernel.

The effect of the resolution model on the iterative reconstruction is evaluated by measuring contrast to noise ratio (CNR) of spherical micro-calcifications in a homogeneous background. After this, our new reconstruction method is compared to the optimized filtered backprojection method for the considered system, by performing two observer studies: the first study simulates clusters of spherical micro-calcifications in a power law background for a free search task; the second study simulates smooth or irregular micro-calcifications in the same type of backgrounds for a classification task.

Results: Including the resolution model in the iterative reconstruction methods increases the CNR of micro-calcifications. The first observer study shows a significant improvement in detection of micro-calcifications $(\mathrm{p}=0.029)$ while the second study shows that performance on a classification task remains the same $(\mathrm{p}=0.935)$ compared to the filtered backprojection method.

Conclusions: Our method shows higher CNR and improved visualization of micro-calcifications in an observer experiment on synthetic data. Further study of the negative results of the classification task showed performance variations throughout the volume linked to the changing noise structure introduced by the combination of the resolution model and the smoothing prior.

\section{INTRODUCTION}

Early detection of breast cancers by mammography has been shown to improve patient outcome [1]. However, some lesions, like masses in dense breasts, remain difficult to detect due to the amount of anatomical noise [2]. A three dimensional imaging technique, like digital breast tomosynthesis (DBT), may be able to solve this problem by removing interference from overlapping dense tissue [3]. Current experience shows that visualization of masses is much improved [4], but problems remain for micro-calcifications $[5,6]$.

One path to improve visualization of microcalcifications is to optimize the dose and angular distribution of the limited angle set of projections [7-9]. These projections are then usually reconstructed with filtered backprojection (FBP) although this reconstruction by FBP is not always optimal $[10,11]$ because of the limited angular range and low dose acquisitions,

\footnotetext{
* koen.michielsen@uzleuven.be
}

especially for small angular range [12]. This while according to $\mathrm{Lu}[9]$ a small angular range is actually better for visualizing subtle micro-calcifications when using a simultaneous algebraic reconstruction technique (SART), showing that reconstruction technique and acquisition geometry are linked and that an optimal geometry for one reconstruction might not be valid for another method.

The second approach is improving and optimizing the reconstruction algorithm itself. Das [13] shows that switching to a penalized maximum likelihood method significantly improves the detection of micro-calcifications. Within one iterative method, the precise choice of parameters of the regularization can make a difference [14], both positive and negative. Therefore $\mathrm{Lu}$ [15] creates an optimized regularization that preserves the contrast of micro-calcifications in DBT and later introduces a multiscale regularization that also preserves details in low contrast lesions [16].

Since iterative reconstruction is most effective when the projection is based on an accurate model of the acquisition process, it might be worth focusing on this aspect in addition to the work on image regularization. Chung 
[17] improves the model by taking the polychromatic nature of the x-ray spectrum into account. However, we believe that next to the limited angular range, the motion of the x-ray tube focal spot during image formation is the major deteriorating factor in many tomosynthesis systems. Tube motion may contribute blurring on a scale several times larger than the pixel spacing on mammography flat panel detectors (typically $\leq 100 \mu \mathrm{m}$ ). This deterioration can be seen as a large drop of the modulation transfer function (MTF) as a function of the height above the detector plane [18].

Resolution recovery methods have been used successfully in iterative reconstruction methods for single photon emission computed tomography (SPECT) [19, 20], positron emission tomography (PET) [21, 22] and computed tomography (CT) $[23,24]$. The general idea behind these methods is that by taking into account the size of the x-ray source size, collimation effects and detector characteristics, the final reconstruction is improved. We will follow the same idea by modeling the combined effect of the x-ray source motion and the finite detector size as a position dependent point spread function (PSF) during reconstruction. Effects from the finite size of the voxels in the reconstructed volume are avoided by using distance driven projection and backprojection [25].

We will combine our resolution model with the noise and acquisition models of the Maximum Likelihood for Transmission (MLTR) algorithm [26, 31], but this algorithm (like all iterative methods) is quite slow in comparison to FBP. Therefore we try to improve the convergence speed of the algorithm by applying a grouped coordinate ascent (GCA) approach [27, 28], where groups of voxels are updated sequentially instead of simultaneously. By choosing the reconstruction planes parallel to the detector as the groups in the GCA algorithm, we can introduce a resolution model which is dependent on the height above the detector. The adapted MLTR algorithm will also be combined with a regularization term to create a maximum a posteriori algorithm (MAPTR).

\section{MATERIALS AND METHODS}

\section{A. Resolution Model}

Iterative reconstruction requires a mathematical model of the image acquisition. In the simplest case the estimated transmission scan $\hat{y}_{i}$ can be written as

$$
\hat{y}_{i}=b_{i} e^{-\sum_{j} l_{i j} \mu_{j}}
$$

with $\mu_{j}$ the linear attenuation in voxel $j$ of the reconstruction volume, $b_{i}$ the blank value for projection line $i$ and $l_{i j}$ the intersection length between projection line $i$ and voxel $j$. In this case we want to capture resolution effects both from the detector and from the pulsed exposures from a continuously moving x-ray source. Equation
(2) expands (1) to include these effects.

$$
\hat{y}_{s \theta}=\int_{\theta-\frac{\alpha}{2}}^{\theta+\frac{\alpha}{2}} \sum_{n} A_{s n} b_{n}(\phi) e^{-\sum_{p} \sum_{k} l_{n k p}(\phi) \mu_{k p}} d \phi
$$

The two-dimensional version of this geometry is shown in figure 1. Index $i$ of the projection lines has been split in detector coordinate $s$ in the XY plane and angle $\theta$ on the arc of projection angles $\phi$, with the integral over these angles $\phi$ representing the tube motion during one of the acquisitions of the tomosynthesis series. Volume coordinate $j$ has been split in plane number $p$ on the Z-axis and in-plane coordinate $k$. The blank value $b_{n}(\phi)$ now includes the variable intensity (waveform) of the x-ray exposure during each pulse and the smoothing kernel with coefficients $A_{s n}$ represents the intrinsic detector blurring.

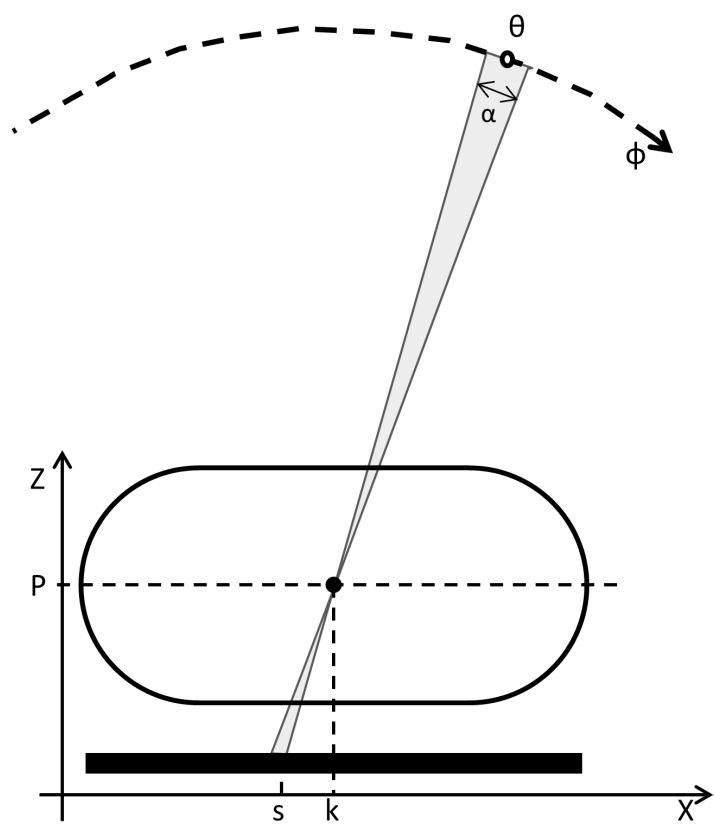

FIG. 1. Acquisition geometry with moving x-ray source.

It is however not feasible to create an update equation from the new acquisition model in equation (2). Therefore we create a simplified version from which to derive the new update equation. 


$$
\begin{aligned}
\hat{y}_{s \theta} & =\sum_{\phi=\theta-\frac{\alpha}{2}}^{\theta+\frac{\alpha}{2}} \sum_{n} A_{s n} b_{n \phi} e^{-\sum_{p} \sum_{k} l_{n \phi k p} \mu_{k p}} \\
& =\sum_{\phi=\theta-\frac{\alpha}{2}}^{\theta+\frac{\alpha}{2}} \sum_{n} A_{s n} b_{n \phi} e^{-\sum_{p \neq P} \sum_{k} l_{n \phi k p} \mu_{k p}} \\
& e^{-\sum_{k} l_{n \phi k P} \mu_{k P}} e^{-\sum_{p \neq P} \sum_{k} l_{s \theta k p} \mu_{k p}} \\
& \approx b_{s \theta} e^{-\sum_{p \neq P} \sum_{k} l_{s \theta k p} \mu_{k p}} A_{s n} \sum_{\phi=\theta-\frac{\alpha}{2}} w_{\theta \phi} e^{-\sum_{k} l_{n \phi k P} \mu_{k P}} \\
& \sum_{n} A_{s n} \sum_{\xi} w_{n \xi}^{P} e^{-\sum_{k} l_{\xi \theta k P} \mu_{k P}} \\
= & b_{s \theta} e^{-\sum_{p \neq P} \sum_{k} l_{s \theta k p} \mu_{k p}} \sum_{n} A_{s n}^{P} e^{-\sum_{k} l_{n \theta k P} \mu_{k P}}
\end{aligned}
$$

Equation (3) is obtained from (2) by replacing the integral over $\phi$ by a sum. In (4) we assume that the volume is homogeneous everywhere except for a few high contrast lesions in plane $P$ and thus split out the contribution of attenuation in that plane. The contributions of the homogeneous parts of the volume are then placed outside of the smoothing kernel in equation (5), with $b_{s \theta}$ the total blank value for angle $\theta$ and $w_{\theta \phi}$ the normalized waveform of the exposure at angle $\theta$. The key approximation is then applied in (6) where the sum over the sparsely sampled angles with kernel $w_{\theta \phi}$ is replaced by a sum over the densely sampled detector pixels with kernel $w_{n \xi}^{P}$, which is combined with kernel $A_{s n}$ to form $A_{s n}^{P}$ in $(7)$.

At this point we can assume that there are more high contrast lesions in the volume, but that they don't overlap in the projections. Repeating the previous steps for all other planes results in (8). Reverting back to index $i$ for the projection lines and index $j$ for the reconstruction volume and including scatter term $s_{i}$ gives our final approximation of the acquisition process in equation (9).

$$
\begin{aligned}
\hat{y}_{s \theta} & \approx b_{s \theta} \prod_{p} \sum_{n} A_{s n}^{p} e^{-\sum_{k} l_{n \theta k p} \mu_{k p}} \\
\hat{y}_{i} & =b_{i} \prod_{p} \sum_{n} A_{i n}^{p} e^{-\sum_{j \in p} l_{n j} \mu_{j}}+s_{i}
\end{aligned}
$$

In essence, the motion blur, which is dependent on the height above the detector plane, is combined with the detector blur to form a system resolution model $A_{i n}^{p}$.

\section{B. Grouped Coordinate Ascent Algorithm for DBT}

In the MLTR algorithm, attenuation distribution $\vec{\mu}$ is obtained by maximizing log-likelihood function $L$. As- suming that the data are subject to Poisson noise, the log-likelihood can be written as

$$
L=\sum_{i} y_{i} \ln \hat{y}_{i}-\hat{y}_{i}
$$

with $y_{i}$ the measured transmission scan, $i$ the index of the projection line and $\hat{y}_{i}$ the mathematical model of the transmission scan, such as for example the models in equations (1) or (9).

With the model in equation (1), we can construct the update step of a gradient ascent algorithm [26, 27, 29] using formula (11), where $\alpha_{j} \geq 0$ is a design parameter.

$$
\begin{aligned}
\Delta \mu_{j} & =\frac{-\alpha_{j} \frac{\partial L}{\partial \mu_{j}}}{\sum_{k} \alpha_{k} \frac{\partial^{2} L}{\partial \mu_{j} \partial \mu_{k}}} \\
& =\frac{\alpha_{j} \sum_{i} l_{i j}\left(y_{i}-\hat{y}_{i}\right)}{\sum_{i} l_{i j} \hat{y}_{i} \sum_{k} \alpha_{k} l_{i k}}
\end{aligned}
$$

Choosing $\alpha_{j}=1$ for all $j$ in equation (12) results in the MLTR algorithm and $\alpha_{j}=\mu_{j}^{\text {old }}+\epsilon$, with $\epsilon$ a small positive constant to make sure $\alpha_{j}>0$, gives the convex algorithm [30]. The computational cost of this algorithm is dominated by the four (back)projections. If $\alpha_{j}$ is constant one of the projections can be precomputed, reducing the cost to three (back)projections per iteration.

To create a GCA algorithm, the image is divided into regions (patches) that are updated separately and sequentially $[27,29]$. Accelerated convergence is partly due to the sequential updates but mainly due to an increased step size in the update. In equation (12) we can consider a patch update as an update with $\alpha_{j}=0$ everywhere except in the current group. Therefore the sum $\sum_{h} \alpha_{h} l_{i h}$ in the denominator will be smaller and the step size for updates will be larger for smaller patches. We choose to use each plane (parallel to the detector surface) in the reconstruction volume as a separate patch. This is both the logical choice, since this is how tomosynthesis images are visualized, and close to optimal, since it minimizes the denominator in equation (12), indicating that voxels in one plane share little information in the projection. Splitting the updates in this way does not change the computational cost because for each iteration the same number of intersection lengths $l_{i j}$ need to be calculated. In the real implementation, this introduces some overhead, but the three full (back)projections in each iteration remain dominant.

The MLTR and MAPTR algorithms combined with the plane-by-plane GCA updates will be called MLTR-p and MAPTR-p from now on.

According to the central slice theorem, a (parallel beam) projection provides a central slice perpendicular to the projection lines in the Fourier domain. It follows that in tomosynthesis, no samples along the vertical axis in the Fourier domain are obtained. These samples correspond to attenuation distributions that are nearly uniform within the plane, but vary in the direction orthogonal to the plane. As a consequence, the maximum 
likelihood algorithm has no strong preference about how attenuation components, that are uniform in the plane, should be distributed along the planes. This is very obvious when choosing the groups as planes parallel to the detector surface, where the patchwork algorithm tends to accumulate all low frequency information in the first updated plane. To ensure that low frequency information will be uniformly distributed over all planes, we initialize the reconstruction volume with a rough estimate of the attenuation and divide the update step for each plane in the first two iterations by the number of planes that still need to be updated in the current iteration. This still leaves a limited non-uniformity in the volume, which is largely removed by reversing the update order of the patches in the second iteration. The effects of this initialization on the reconstruction of a homogeneous phantom are shown in figure 2.

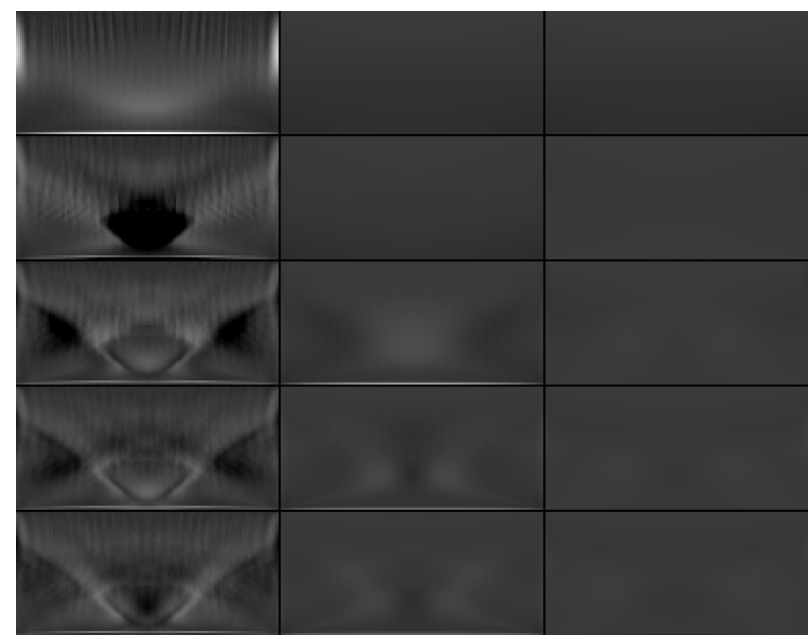

FIG. 2. Effect of initial updates on the patchwork reconstruction of a homogeneous phantom. Axial slices of the first five iterations (rows) are shown. Left: normal (full weight) updates, going from bottom to top; middle: reduced weight updates in iterations 1 and 2, going from bottom to top; right: reduced weight updates in iterations 1 and 2, going from bottom to top, except for iteration 2, where the update direction is reversed.

\section{Patchwork Reconstruction with Resolution Modeling}

To create the full patchwork reconstruction, we combine the resolution model with the grouped coordinate ascent updates so that each group (patch) has its own unique acquisition model determined by the resolution model and is updated separately and sequentially [31].

To derive the update for the new algorithm, we introduce the following definitions that describe the acquisi- tion model.

$$
\begin{aligned}
\hat{y}_{i} & =b_{i} \prod_{p} \bar{\psi}_{i}^{p}+s_{i} \\
\bar{\psi}_{i}^{p} & =\sum_{n} A_{i n}^{p} \psi_{n}^{p} \\
\psi_{i}^{p} & =e^{-\sum_{j \in p} l_{i j} \mu_{j}}
\end{aligned}
$$

With this we can calculate the update step in using equation (11), where $\alpha_{j}>0$ inside the current patch and $\alpha_{j}=0$ outside. The approximation for the second derivative comes from the assumption that the intersection lengths are smooth on the scale of the kernel $A_{i n}^{p}$ (18).

$$
\begin{gathered}
-\alpha_{j} \frac{\partial L}{\partial \mu_{j}}=\alpha_{j} \sum_{i} l_{i j} \psi_{i}^{p(j)} \sum_{n} A_{i n}^{p(j)} \frac{y_{n}-\hat{y}_{n}}{\bar{\psi}_{n}^{p(j)}} \frac{\hat{y}_{n}-s_{n}}{\hat{y}_{n}} \\
\sum_{k} \alpha_{k} \frac{\partial^{2} L}{\partial \mu_{j} \partial \mu_{k}} \approx-\sum_{i} l_{i j} \psi_{i}^{p(j)} \cdot \sum_{k} \alpha_{k} l_{i k} \cdot \\
\sum_{n} A_{i n}^{p(j)} \frac{\hat{y}_{n}-s_{n}}{\bar{\psi}_{n}^{p(j)}}\left(1-\frac{y_{n} s_{n}}{\hat{y}_{n}^{2}}\right) \\
\sum_{n} A_{i n}^{p} \psi_{n}^{p} \sum_{k \in p} l_{n k} \approx \sum_{k \in p} l_{i k} \sum_{n} A_{i n}^{p} \psi_{n}^{p}
\end{gathered}
$$

If $\alpha_{j}=\mu_{j}$ or constant for all $j$ inside the current patch, then this update equation has a computational cost of five (back)projections for each full iteration. The convolution operation adds an additional cost, but this is small in comparison to the additional forward and backprojection needed in this algorithm.

The MLTR-p and MAPTR-p algorithms that include the plane-by-plane resolution model will be called MLTRpr and MAPTR-pr from now on.

\section{Determining Smoothing Kernel $A_{i n}^{p}$.}

To determine the smoothing kernels $A_{i n}^{p}$ we assume that the exposure waveform is Gaussian, and the pulse duration in the DICOM header represents its full width at half maximum (FWHM). This FWHM is then projected from the arc on which the focal spot moves onto the detector plane with projection lines crossing in the midpoint of plane $p$, as shown in figure 1. It is clear from the geometry that the projected FWHM will be smaller if plane $p$ is closer to the detector, and wider if $p$ is further from the detector.

Figures 3 and 4 show that these approximations are appropriate for small calcification with the relative error in the projection increasing with the diameter (and thus contrast) of the projected object.

Figure 3 shows the projections of five calcifications with diameters 100, 125, 150, 175 and $200 \mu \mathrm{m}$, located at 
$60 \mathrm{~mm}$ above the detector plane. The left column shows the projections created using a simulation according to the accurate description in formula (3) and the middle column is simulated using the approximated description in formula (9). The right column then shows the relative error in the projection by dividing both projections. The maximum error of the projections without resolution model (1) and with resolution model (9) are shown in figure 4.

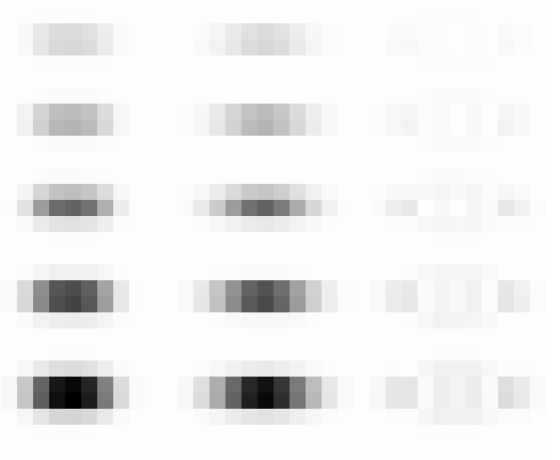

FIG. 3. Projection of 5 calcifications (diameters: 100, 125, 150,175 and $200 \mu \mathrm{m}$ ) located at $60 \mathrm{~mm}$ above the detector. Left column: simulation using formula 3 ; middle column: projection using formula 9 ; right column: error in the projection by dividing the left and middle columns.

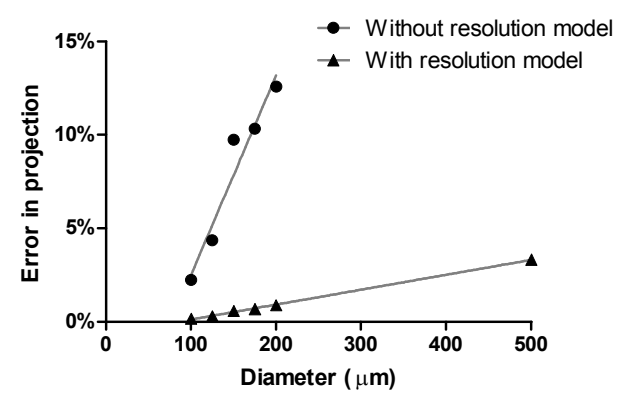

FIG. 4. Maximum relative error for projections of different diameter calcifications without resolution model (equation (1)) and with the approximate model (equation (9)).

\section{E. Phantom Simulation and Reconstruction}

To test the new reconstruction method, we compare it to other methods by evaluating reconstructions of phantoms containing simulated lesions. The creation and simulation of these phantoms is described in this section, the evaluation methods in the next section.

We simulated two types of background images: a simple homogeneous background with linear attenuation set at a 50-50 mix of adipose and glandular tissue and a background with structured noise created by filtering white noise with a power law filter $[32,33] f(\nu)=\kappa / \nu^{\beta}$, with $\nu$ the frequency, $\beta=3$ and $\kappa=10^{-5} \mathrm{~mm}^{-1}$. The linear attenuation coefficients of this background were rescaled to values between the attenuation of adipose and glandular tissue. The resulting images were reduced to $500 \times 500 \times 200$ isotropic voxels with sides of $85 \mu \mathrm{m}$. Figure 5 shows some examples of the second type of background. These volumes are then placed in one of three possible locations, always with one side above the chest-side detector edge: central at $27 \mathrm{~mm}$ above the detector plane (location 1), $75 \mathrm{~mm}$ off center at a height of $47 \mathrm{~mm}$ (location 2) and central at $67 \mathrm{~mm}$ above the detector plane (location 3 ). These locations are shown on figure 6 .

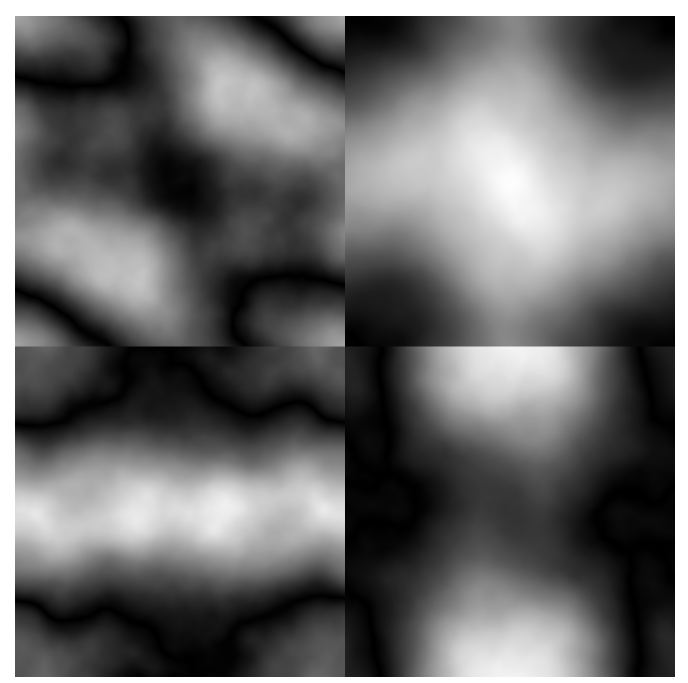

FIG. 5. Four examples of the power law backgrounds.

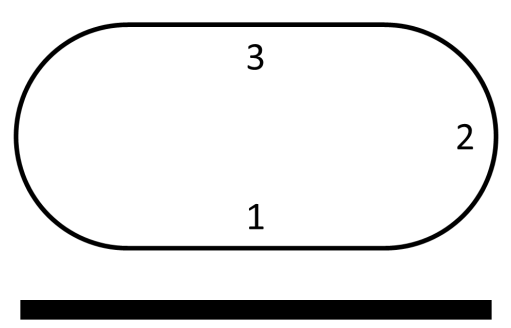

FIG. 6. Three locations in the breast where the simulated backgrounds are located.

Sixteen spherical micro-calcifications were added to the middle plane of the homogeneous background in a four by four grid. Separate volumes were created for calcifications with a diameter of $100 \mu \mathrm{m}, 150 \mu \mathrm{m}$ and $200 \mu \mathrm{m}$.

The structured background images were used to generate two additional data sets: in the first we added a random number of clusters to each background image, Poisson distributed with a mean of 1.0 per image, and placed at a random location within the volume (but not on the 
edge). Each cluster consisted of a random number of calcifications, with a mean of 2.5 per cluster (again Poisson distributed), but with a minimum of a single calcification per cluster. The individual calcifications were spherical, with a diameter between 100 and $200 \mu \mathrm{m}$, spaced 0.5 to $1.5 \mathrm{~mm}$ apart in a random direction and set in a volume with isotropic voxel spacing of $5 \mu \mathrm{m}$. Figure 7 shows some enlarged examples of the generated clusters.

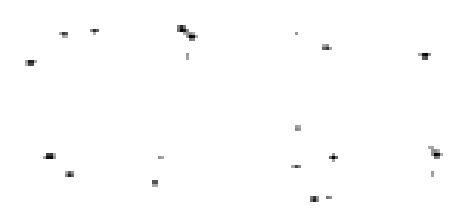

FIG. 7. Eight examples of simulated clusters consisting of spherical micro-calcifications.

For the second data set using the structured backgrounds, we created two series of micro-calcifications (smooth, corresponding to Le Gal II and irregular, corresponding to Le Gal IV) according to the method of Näppi [34]. These micro-calcifications were rescaled to a diameter of $200,300,400,500$ or $600 \mu \mathrm{m}$ by changing the voxel spacing of the simulated volume, resulting in isotropic voxel spacings between 6 and $18 \mu \mathrm{m}$, depending on the rescaling. Figure 8 shows some enlarged examples of the generated calcifications.

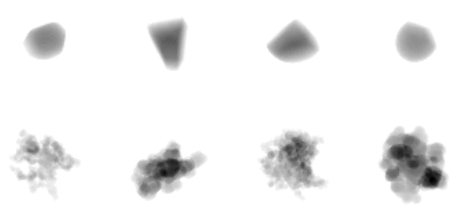

FIG. 8. Examples of smooth (Le Gal II, top row) and irregular (Le Gal IV, bottom row) micro-calcifications.

Projections of these volumes were simulated according to the acquisition model described in equation (3) and with increased detector sampling. Nine source positions were sampled for each exposure angle, corresponding to an exposure time of $120 \mathrm{~ms}$ per projection, x-ray energy was set to $20 \mathrm{keV}$ and Poisson noise was generated with a blank scan of 1500 photons per pixel (12.5 $\mu$ Gy detector dose after attenuation).

The geometric blurring parameters for our model were determined for the Mammomat Inspiration system (Siemens, Erlangen, Germany - Breast tomosynthesis with Siemens MAMMOMAT Inspiration is an investigational practice and is limited by U.S. law to investigational use. It is not commercially available in the U.S. and its future availability cannot be ensured.), which is in clinical use on site. Therefore we included the Siemens iFBP method [35] as an additional point of reference next to the iterative methods we described. It was used without detector binning, with slice thickness filter and with a filter designed so that resulting reconstructed slices resemble 2D mammography images.

For the maximum a posteriori methods, the Huber prior (equation (19)) was used, with $\beta=3.0 \cdot 10^{-4}$ and $\delta=2.5 \cdot 10^{-4} \mathrm{~mm}^{-1}$. Since the average reconstructed attenuation is about $0.06 \mathrm{~mm}^{-1}$, the prior function is mostly active in linear mode (second line of equation (19)).

$$
\begin{aligned}
& \left|\mu_{j}-\mu_{k}\right|<\delta: P(\vec{\mu})=-\beta \sum_{j, k} w_{j k} \frac{\left(\mu_{j}-\mu_{k}\right)^{2}}{2 \delta^{2}} \\
& \left|\mu_{j}-\mu_{k}\right| \geq \delta: P(\vec{\mu})=-\beta \sum_{j, k} w_{j k} \frac{\left|\mu_{j}-\mu_{k}\right|-\delta / 2}{\delta}
\end{aligned}
$$

Figure 9 shows an example of three of these reconstruction methods (iFBP, MLTR-pr and MAPTR-pr) for two simulated calcifications.

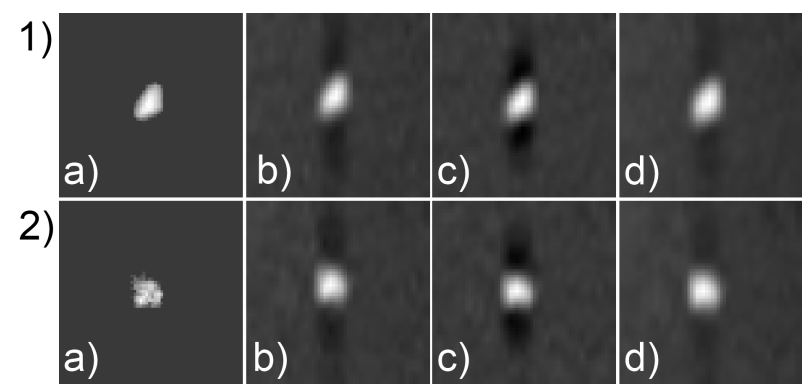

FIG. 9. Projection and reconstruction of two simulated calcifications. 1) smooth (Le Gal II), 2) irregular (Le Gal IV); a) high resolution projection, b) Siemens iFBP, c) MLTR-pr, d) MAPTR-pr.

\section{F. Reconstruction Comparison}

The effect of the resolution model on the iterative reconstruction is shown by evaluating peak contrast to noise ratio (pCNR) for 50 iterations of MLTR-p, MLTRpr, MAPTR-p and MAPTR-pr, using the first simulated phantom described in section IIE. The pCNR was calculated by dividing the difference of the maximum attenuation in the reconstructed calcification and the median attenuation of a surrounding region of 32 by 32 pixels by the standard deviation in that surrounding region.

We compare the convergence speed of the patchwork iterative methods with and without resolution model (MLTR-p, MLTR-pr, MAPTR-p and MAPTR-pr) with their non-patched equivalents (MLTR and MAPTR) by plotting the difference between the likelihood and the maximum likelihood $\left(L-L_{\max }\right)$ as a function of iteration number for a reconstruction of the pCNR phantom with $150 \mu \mathrm{m}$ calcification placed in region 3 used for the 
pCNR measurements described above. Likelihood $L$ is defined in equation (10) and the maximum value of the likelihood $L_{\max }$ can be calculated by replacing $\hat{y}_{i}$ with $y_{i}$ in this equation. These first results were then used to determine the number of iterations to use for our algorithms in further evaluation.

A final, more detailed comparison was limited to the MLTR-pr, MAPTR-pr and iFBP reconstructions. It was split in two distinct observer experiments: first, a free search study to check the detectability of small spherical micro-calcifications and second, a classification task to check the discrimination between smooth (Le Gal II) and irregular (Le Gal IV) micro-calcifications.

For the detection study, 7 readers performed a free search on 120 cases for each reconstruction (with 40 of those cases used for initial training) and scored detected lesions on a 4 point scale as shown in table I. Results were analyzed using the weighted JAFROC method [36].

\begin{tabular}{cl} 
Score & Description \\
\hline 1 & I see a hint of a calcification \\
2 & This might be a calcification \\
3 & This is probably a calcification \\
4 & I am sure this is a calcification
\end{tabular}

TABLE I. Evaluation scale for the detection experiment.

For the classification study, 5 readers evaluated 300 cases for each reconstruction (of which 100 cases were used as initial training) by classifying them as smooth or irregular and providing their certainty of this classification (low, medium or high certainty). These results were transformed to scores from 1 (smooth, high certainty) to 6 (irregular, high certainty) and then analyzed using the DBM MRMC method [37].

Data of the classification study was further split per location and in overlapping size groups $(200-400 \mu \mathrm{m}$, $300-500 \mu \mathrm{m}$ and $400-600 \mu \mathrm{m}$ ) for further analysis using the same methods as the full data set.

\section{RESULTS}

Figure 10 shows the mean pCNR for each calcification diameter (100, 150 and $200 \mu \mathrm{m}$ ) and each location (as shown in figure 6 ). The reconstructions that include a resolution model score equal or higher than those without resolution model, and maximal pCNR is achieved by the MAPTR-pr algorithm before iteration 10.

The reconstructions shown in figure 11 (iFBP and 50 iterations of both MLTR-p and MLTR-pr on the projections in figure 3 ) illustrate that the resolution model has a significant effect on the FWHM and intensity in the reconstruction. The FWHM and contrast is similar between the iFBP reconstruction and the MLTR-p algorithm, but there are no artifacts visible in the MLTR-p reconstruction. The MLTR-pr reconstruction shows both improved FWHM and contrast.
Figure 12 shows $L-L_{\max }$ in function of the logarithm of the number of iterations for the MLTR, MLTR-p and MLTR-pr reconstructions. The algorithms that include a regularization term (MAPTR, MAPTR-p and MAPTRpr) are not shown on the graph for clarity, since at this scale the curves would overlap. The MLTR and MLTRp reconstructions converge to the same likelihood, while the MLTR-pr converges to a different value at a slower pace due to the resolution model. Some concrete comparisons of the convergence speed: MLTR-p only needs 3 iterations to reach the likelihood that MLTR reaches in iteration 25, 7 iterations to reach the likelihood of 100 iterations of MLTR and 23 iterations to reach 500 iterations of MLTR.

This can also be seen subjectively in figure 13 . The images on the top row, 20 iterations of MAPTR on the left and 3 iterations of MAPTR-p on the right are almost alike. The images on the bottom row are 3 iterations of the MAPTR algorithms and show that the image intensities have not yet converged. The image on the left is shown with the same window level setting as the images in the top row, while the window level setting for the image on the right is set from the minimum to the maximum pixel value to show more details.

Tables II and III and figure 14 shows results for the detection study. The extension from the point of the lowest confidence score ( 1 in table I) is shown in grey. There are significant differences between the iFBP and the MAPTR-pr reconstruction $(\mathrm{p}=0.029)$ and between the MLTR-pr and MAPTR-pr reconstructions $(\mathrm{p}=0.022)$ for detecting the smallest micro-calcifications $(<200 \mu \mathrm{m})$. There is no difference between the iFBP and the MLTRpr reconstruction $(\mathrm{p}=0.893)$.

\begin{tabular}{l|c} 
Reconstruction & Figure of Merit $(95 \%$ CI $)$ \\
\hline Siemens iFBP & $0.780(0.710-0.851)$ \\
MLTR-pr & $0.778(0.717-0.840)$ \\
MAPTR-pr & $0.819(0.765-0.873)$
\end{tabular}

TABLE II. AFROC figures of merit from the detection study.

\begin{tabular}{l|r} 
Differences & p-value \\
\hline Siemens iFBP vs. MLTR-pr & 0.893 \\
Siemens iFBP vs. MAPTR-pr & 0.029 \\
MLTR-pr vs. MAPTR-pr & 0.022
\end{tabular}

TABLE III. P-values for the differences between the figures of merit from the detection study.

\begin{tabular}{l|c} 
Reconstruction & Figure of Merit $(95 \%$ CI $)$ \\
\hline Siemens iFBP & $0.774(0.733-0.815)$ \\
MLTR-pr & $0.773(0.716-0.829)$ \\
MAPTR-pr & $0.769(0.719-0.819)$ \\
\hline \hline p-value & 0.935
\end{tabular}

TABLE IV. ROC figures of merit from the classification study. 
Location 1
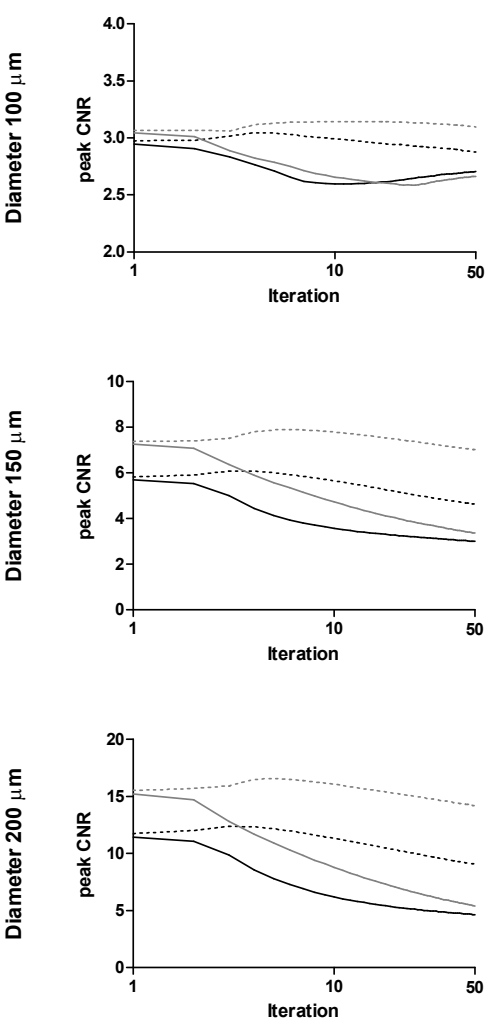

- MLTR_p
Location 2
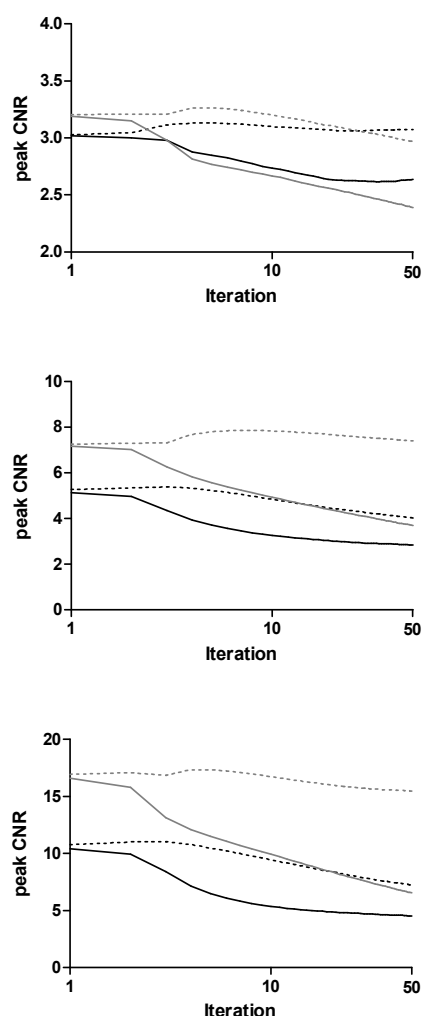

$\cdots$ MAPTR_p - MLTR_pr
Location 3
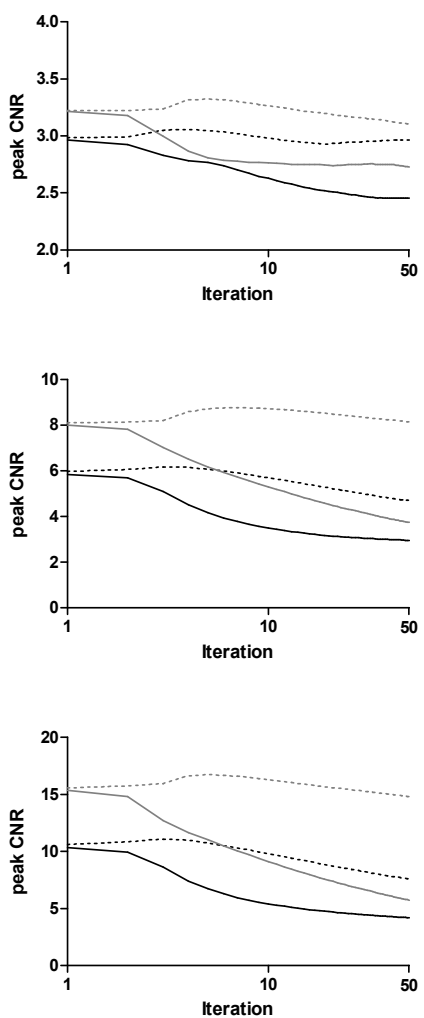

MAPTR_pr

FIG. 10. Overview of pCNR results.

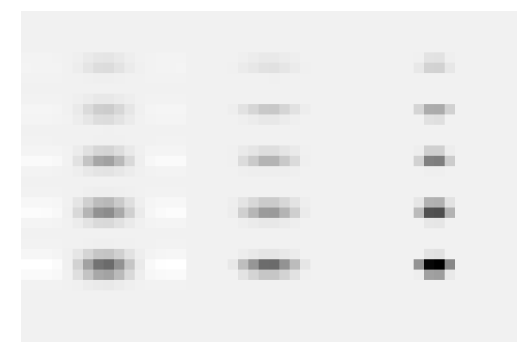

FIG. 11. Reconstruction of the simulated projection shown in figure 3. Left: Siemens iFBP; middle: 50 iterations of MLTR-p; right: 50 iterations of MLTR-pr.

Table IV and figure 15 show the results for the shape discrimination study with the area under the ROC curve as the figure of merit. The p-value of 0.935 indicates that the three reconstruction methods have identical performance when considering shape discrimination of small lesions.

The results from the further sub-analysis of the classification task are listed in table V. None of the results in this table are statistically significantly different $(\mathrm{p}>$ $0.05)$.

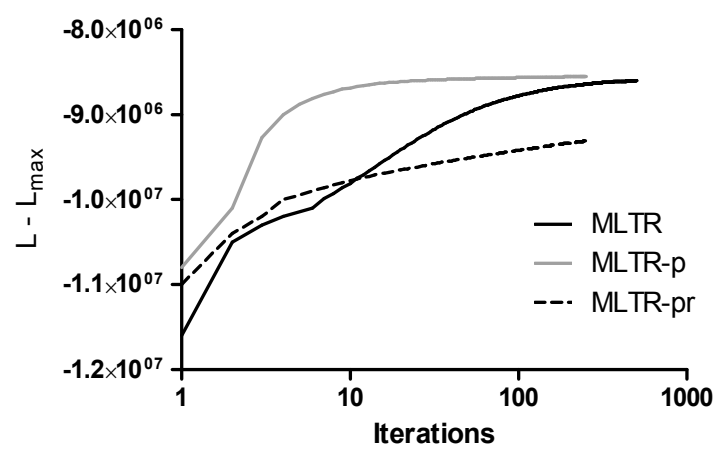

FIG. 12. Convergence of the MLTR, MLTR-p and MLTR-pr algorithms.

\section{DISCUSSION}

We introduced a resolution model in the grouped coordinate ascent algorithm to improve visualization of micro-calcifications. The first evaluation shows the effect of the resolution model on the GCA algorithm (MLTRpr and MAPTR-pr vs MLTR-p and MAPTR-p). The increased pCNR when applying the resolution model can be explained by the fact that the attenuation of the calcifications is now concentrated in less voxels so that the 

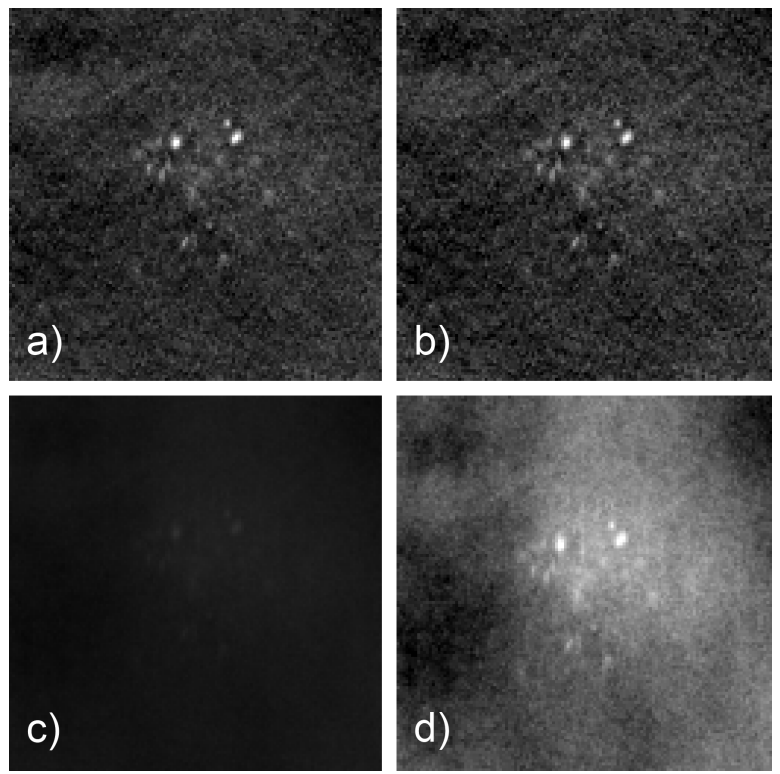

FIG. 13. A cluster of micro-calcifications in the reconstructed ROI to evaluate Convergence. a) 20 iterations of MAPTR; b) 3 iterations of MAPTR-p; c) 3 iterations of MAPTR; d) 3 iterations of MAPTR with adjusted window level.

\begin{tabular}{c|l|lll} 
Location & Reconstruction & \multicolumn{3}{|c}{ Figure of Merit } \\
& & small & medium & large \\
\hline \hline \multirow{3}{*}{1} & Siemens iFBP & 0.691 & 0.789 & 0.842 \\
& MLTR-pr & 0.681 & 0.779 & 0.839 \\
& MAPTR-pr & 0.726 & 0.803 & 0.844 \\
\hline \multirow{3}{*}{2} & Siemens iFBP & 0.633 & 0.684 & 0.796 \\
& MLTR-pr & 0.687 & 0.756 & 0.822 \\
& MAPTR-pr & 0.620 & 0.702 & 0.858 \\
\hline \multirow{3}{*}{3} & Siemens iFBP & 0.685 & 0.779 & 0.870 \\
& MLTR-pr & 0.734 & 0.811 & 0.859 \\
& MAPTR-pr & 0.652 & 0.754 & 0.827 \\
\hline
\end{tabular}

TABLE V. ROC figures of merit of the classification study sub-analyses.

contrast increases, and that the resolution model causes an additional smoothing of the background, thus reducing the noise. The higher pCNR of the regularized versions of the algorithms is a result of the Huber prior which reduces noise in the background while maintaining the contrast in the calcifications for these reconstructions.

Further evaluations then show that the patchwork reconstruction with resolution modeling and smoothing prior (MAPTR-pr) can improve upon the Siemens iFBP after only 3 iterations for detecting very small microcalcifications while performing at the same level for classifying slightly larger micro-calcifications.

The identical performance of all three reconstruction methods for the classification task was unexpected, so we examined the scores further by splitting up the results per lesion size and location (shown in table V). In this table, we can see that for location 1 MAPTR-pr has the highest scores and for locations 2 and 3 MLTRpr performs better than the two other reconstructions. We believe this is caused by the smoothing prior, which smooths more in planes further from the detector, because the likelihood provides less information there. A possible solution which we will investigate, is to vary the prior strength as a function of the position, aiming at a more uniform balance between prior and likelihood [38].

A useful feature of the proposed algorithm is the improved convergence speed per iteration that comes from applying a grouped coordinate ascent algorithm, although this effect is reduced by the resolution model. The convergence of the MLTR-pr algorithm towards a lower likelihood than MLTR-p is a consequence of the resolution model which strongly reduces the degrees of freedom of the reconstruction. This reduction limits the propagation of Poisson noise and thus lowers the similarity between the measured data and the estimated transmission scan, and therefore the likelihood of the reconstruction. Nevertheless, convergence of the MLTR-pr and MAPTRpr algorithms is sufficiently fast to make the computation time acceptable for clinical applications.

From the pCNR results in figure 10 we expect that a few more iterations will further improve the results, but we would like to keep reconstruction time as short as possible. Therefore we will investigate more effective initialization methods, that allow full weight updates in the two first iterations where we now used reduced strength updates to allow for a simple homogeneous initialization.

The new algorithm currently results in a limited improvement on the clinical image quality, as shown in a comparison with iFBP in figure 16. This makes sense when considering the fact that the iFBP algorithm has been specifically optimized for the Mammomat Inspiration system. We expect further improvements in our algorithm when including the measured point spread functions of the system in the resolution model instead of a Gaussian approximation.

\section{CONCLUSIONS}

The described method greatly increases convergence rate per iteration of DBT reconstruction while including an accurate resolution model. Adding a Huber-prior to the algorithm limits the noise in the image and allows reconstruction of clinical images in only 3 iterations while increasing detection performance in comparison to iFBP and maintaining the same level of lesion discrimination performance.

\section{ACKNOWLEDGMENTS}

The authors would like to thank Nicholas Marshall for providing the MTF measurements for the resolution model; Federica Zanca for her advice on designing the 


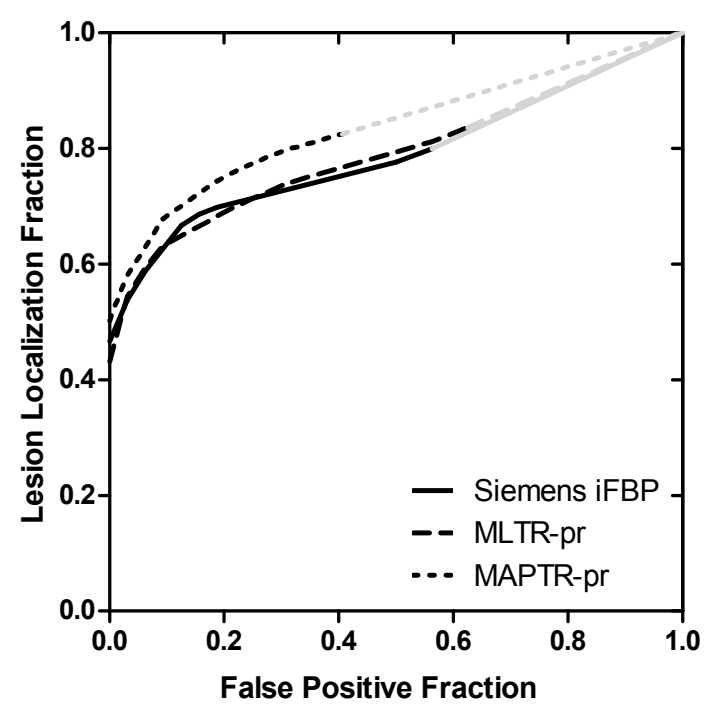

FIG. 14. AFROC curves from the detection study results.

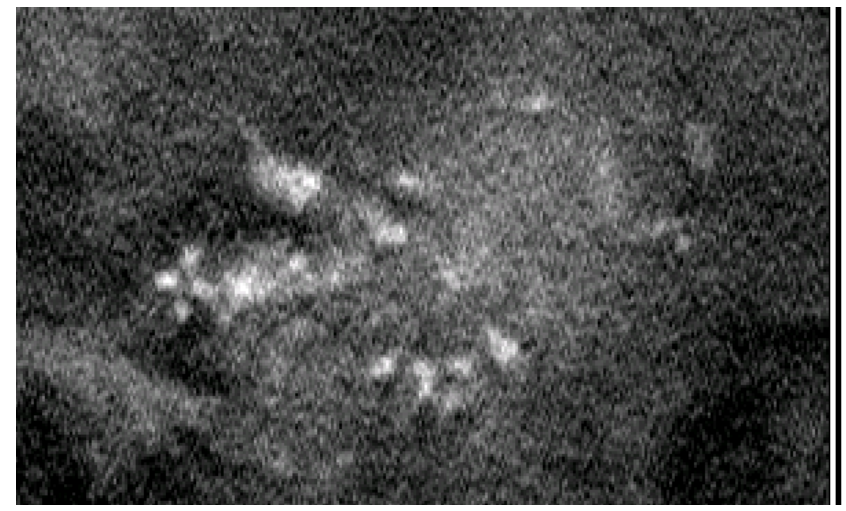

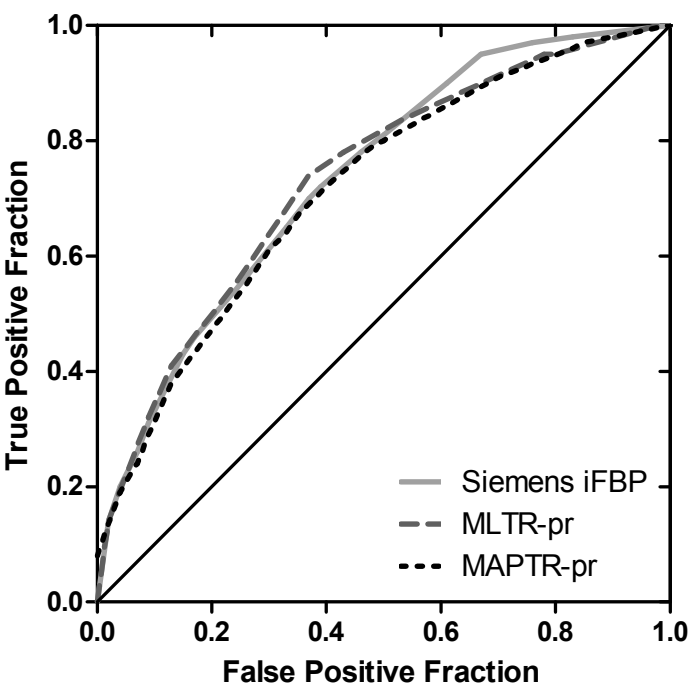

FIG. 15. ROC curves from the classification study results.

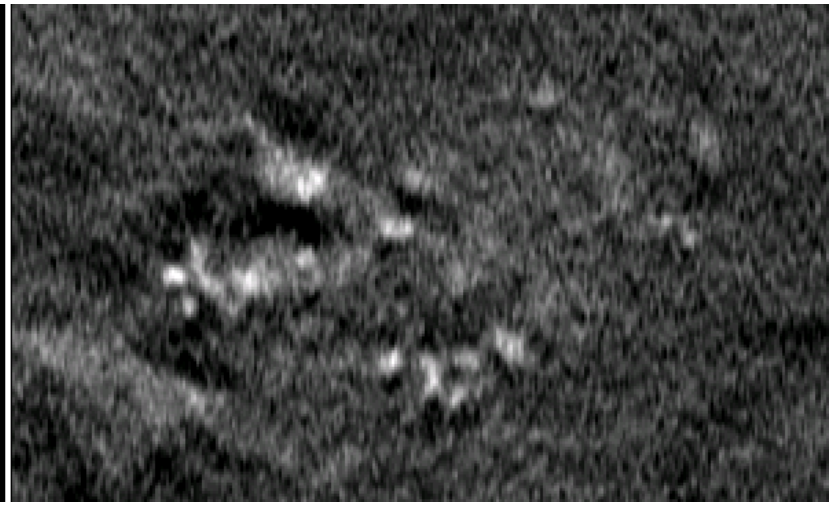

FIG. 16. A cluster of micro-calcifications (left: iFBP, right: three iterations of MAPTR-pr).

observer study; and Joke Bints, Gregory Cieters, Lesley Cockmartin, Annelies Jacobs, Xochitl Lopez Rendon, Ahmadreza Rezaei, Elena Salvagnini, Eman Shaheen and Lin Zhou for their participation in the observer study.
This work is supported by Siemens AG and by SBO project QUANTIVIAM (060819) of the Institute for Promotion of Innovation through Science and Technology in Flanders (IWT-Vlaanderen).
[1] L. Nyström, I. Andersson, N. Bjurstam, J. Frisell, B. Nordenskjöld, L.E. Rutqvist, "Long-term effects of mammography screening: updated overview of the Swedish randomised trials," Lancet 359, 909-919 (2002).

[2] R.E. Bird, T.W. Wallace, B.C. Yankaskas, "Analysis of cancers missed at screening mammography," Radiology 184, 613-617 (1992).

[3] J.A. Baker and J.Y. Lo, "Breast tomosynthesis: state-ofthe-art and review of the literature," Acad. Radiol. 18, 1298-1310 (2011).

[4] J.M. Park, E.A. Franken, M. Garg, M.L. Fajardo, L.T. Niklason, "Breast tomosynthesis: present considerations and future applications," RadioGraphics 27 Suppl 1, S231-S240 (2007).
[5] J.T. Dobbins III, "Tomosynthesis imaging: At a translational crossroads," Med. Phys. 36, 1956-1967 (2009).

[6] T.M. Svahn, D.P. Chakraborty, D. Ikeda, S. Zackrisson, Y. Do, S. Mattsson, I. Andersson, "Breast tomosynthesis and digital mammography: a comparison of diagnostic accuracy," Brit. J. Radiol. (published online before print, 2012)

[7] M. Das, H.C. Gifford, J.M. O'Conner, S.J. Glick, "Evaluation of a variable dose acquisition technique for microcalcification and mass detection in digital breast tomosynthesis," Med. Phys. 36, 1976-1984 (2009).

[8] Y. Hu and W. Zhao, "The effect of angular dose distribution on the detection of microcalcifications in digital breast tomosynthesis," Med. Phys. 38, 2455-2466 (2011). 
[9] Y. Lu, H. Chan, J. Wei, M. Goodsitt, P.L. Carson, L.M. Hadjiiski, A. Schmitz, J.W. Eberhard, B.E.H. Claus, "Image quality of microcalcifications in digital breast tomosynthesis: effects of projection-view distributions," Med. Phys. 38, 5703-5712 (2011).

[10] T. Wu, R.H. Moore, E.A. Rafferty, D.B. Kopans, "A comparison of reconstruction algorithms for breast tomosynthesis," Med. Phys. 31, 2636-2647 (2004).

[11] Y. Zhang, H. Chan, B. Sahiner, J. Wei, M.M. Goodsitt, L.M. Hadjiiski, J. Ge, C. Zhou, "A comparative study of limited-angle cone-beam reconstruction methods for breast tomosynthesis," Med. Phys. 33, 3781-3795 (2006).

[12] T. Mertelmeier, J. Ludwig, B. Zhao, W. Zhao, "Optimization of tomosynthesis acquisition parameters: angular range and number of projections" in Digital Mammography, edited by E. Krupinski (Springer Berlin / Heidelberg, 2008), pp. 220-227.

[13] M. Das, H.C. Gifford, J.M. O'Connor, S.J. Glick, "Penalized maximum likelihood reconstruction for improved microcalcification detection in breast tomosynthesis," IEEE Trans. Med. Imaging 30, 904-914 (2011).

[14] E.Y. Sidky, X. Pan, I. Reiser, R.M. Nishikawa, R.H. Moore, D.B. Kopans, "Enhanced imaging of microcalcifications in digital breast tomosynthesis through improved image-reconstruction algorithms," Med. Phys. 36, 49204932 (2009).

[15] Y. Lu, H. Chan, J. Wei, L.M. Hadjiiski, "Selectivediffusion regularization for enhancement of microcalcifications in digital breast tomosynthesis reconstruction," Med. Phys. 37, 6003-6014 (2010).

[16] Y. Lu, H. Chan, J. Wei, L.M. Hadjiiski, C. Zhou, "Multiscale regularized reconstruction for enhancing microcalcification in digital breast tomosynthesis," in Proc. SPIE, 2012.

[17] J. Chung, J.G. Nagy, I. Sechopoulos, "Numerical Algorithms for Polyenergetic Digital Breast Tomosynthesis Reconstruction," SIAM Journal on Imaging Sciences 3, $0-0$ (2010).

[18] N. Marshall and H. Bosmans, "Measurements of system sharpness for two digital breast tomosynthesis systems," Phys. Med. Biol. 57, 7629-7650 (2012).

[19] B.C. Penney, M.A. King, K. Knesaurek, "A projector, backprojector pair which accounts for the twodimensional depth and distance dependent blurring in SPECT," IEEE Trans. Nuc. Sci. 37, 681-686 (1990).

[20] G.L. Zeng, G.T. Gullberg, B.M.W. Tsui, J.A. Terry, "Three-dimensional iterative reconstruction algorithms with attenuation and geometric point response correction," IEEE Trans. Nuc. Sci. 38, 693-702 (1991).

[21] R.H. Huesman, E.M. Salmeron, J.R. Baker, "Compensation for crystal penetration in high resolution positron tomography," IEEE Trans. Nuc. Sci. 36, 1100-1107 (1989).

[22] Z. Liang, "Detector response restoration in image reconstruction of high resolution positron emission tomography," IEEE Trans. Med. Imag. 13, 314-321 (1994).

[23] J.A. Browne, J.M. Boone, T.J. Holmes, "Maximumlikelihood X-ray computed-tomography finite-beamwidth considerations," Appl. Opt. 34, 5199-5209 (1995).

[24] K. Zeng, B. De Man, J.-B. Thibault, Y. Zhou, C. Bouman, K. Sauer, "Spatial resolution enhancement in
CT iterative reconstruction" in Nuclear Science Symposium Conference Record (NSS/MIC), 2009 IEEE, pp. 3748-3751.

[25] B. De Man and S. Basu, "Distance-driven projection and backprojection in three dimensions," Phys. Med. Biol. 49, 2463-2475 (2004).

[26] J. Nuyts, B. De Man, P. Dupont, M. Defrise, P. Suetens, L. Mortelmans, "Iterative reconstruction for helical CT: a simulation study," Phys. Med. Biol. 43, 729-737 (1998).

[27] J. A. Fessler, E.P. Ficaro, N.H. Clinthorne, K. Lange, "Grouped-coordinate ascent algorithms for penalizedlikelihood transmission image reconstruction," IEEE Trans. Med. Imag. 16, 166-175 (1997).

[28] J.A. Fessler and D. Kim, "Axial block coordinate descent (ABCD) algorithm for X-ray CT image reconstruction," in Proceedings of the 11th International Meeting on Fully Three-Dimensional Image Reconstruction in Radiology and Nuclear Medicine, pp. 262-265.

[29] K. Van Slambrouck and J. Nuyts, "Accelerated convergence with image-block iterative reconstruction," in Nuclear Science Symposium Conference Record (NSS/MIC), 2011 IEEE, pp. 2576-2580.

[30] K. Lange and J.A. Fessler, "Globally convergent algorithms for maximum a posteriori transmission tomography," IEEE Trans. Image Process. 4, 1430-1438 (1995).

[31] K. Van Slambrouck and J. Nuyts, "Metal artifact reduction in computed tomography using local models in an image block-iterative scheme," Med. Phys. 39, 7080-7093 (2012).

[32] K.G. Metheany, C.K. Abbey, N. Packard, J.M. Boone, "Characterizing anatomical variability in breast CT images," Med. Phys. 35, 4685-4694 (2008).

[33] E. Engstrom, I.S. Reiser, R.M. Nishikawa, "Comparison of power spectra for tomosynthesis projections and reconstructed images," Med. Phys. 36, 1753-1758 (2009).

[34] J. Näppi, P.B. Dean, O. Nevalainen, S. Toikkanen, "Algorithmic 3D simulation of breast calcifications for digital mammography," Comput. Meth. Prog. Bio. 66, 115-124 (2001).

[35] J. Ludwig, T. Mertelmeier, H. Kunze, W. Harer, "A Novel Approach for Filtered Backprojection in Tomosynthesis Based on Filter Kernels Determined by Iterative Reconstruction Techniques," in Digital Mammography, edited by E. Krupinski (Springer Berling / Heidelberg 2008), pp. 612-620.

[36] D.P. Chakraborty and K.S. Berbaum, "Observer studies involving detection and localization: modeling, analysis, and validation," Med. Phys. 31, 2313-2330 (2004).

[37] D.D. Dorfman, K.S. Berbaum, C.E. Metz, "Receiver operating characteristic rating analysis. Generalization to the population of readers and patients with the jackknife method," Invest. Radiol. 27, 723-731 (1992).

[38] J.A. Fessler and W.L. Rogers, "Spatial resolution properties of penalized-likelihood image reconstruction: spaceinvariant tomographs," IEEE Trans. Image Process. 5, 1346-1358 (1996). 